UDC 517.54

S. Yu. GraF

\title{
ON DISTORTION OF THE MODULI OF RINGS UNDER LOCALLY QUASICONFORMAL MAPPINGS IN $\mathbb{R}^{n}$
}

Abstract. Some of the earlier results of the author conce
distortion of the moduli of ring domains under planar locally
siconformal mappings are generalized on the case of locally
conformal mappings in $\mathbb{R}^{n}, n \geq 2$. The main result of the
represents the sharp double-sided estimation of modulus
of the image $D$ of the concentric spherical ring $K(r, R)=$
$\left.\in \mathbb{R}^{n}: r<|x|<R\right\}$ under locally quasiconformal homeon
phism $f:$
\[ \int_{r}^{R} P_{f}^{1 /(1-n)}(t) \frac{d t}{t} \leq \operatorname{Mod}(D) \leq \int_{r}^{R} P_{f}^{1 /(n-1)}(t) \frac{d t}{t} . \]

Here the function $P_{f}$ is the majorant of dilatation of mapping $f$ and $P_{f}$ is well defined as

$$
P_{f}(t)=\lim _{\varepsilon \rightarrow 0+} \operatorname{essup}\left\{p_{f}(x): t-\varepsilon \leq|x| \leq t+\varepsilon\right\} .
$$

As the consequence of the main inequalities the sharp estimations of the derivative $f^{\prime}(0)$ of the normalized locally quasiconformal automorphisms $f$ of the unit ball in terms of majorant of the dilatation of function $f$ are proved. The sharpness of the results is demonstrated by examples of non-trivial locally quasiconformal mappings with unbounded dilatation that provide equalities in the estimations. The main theorems were obtained by means of method of moduli of families of curves and hypersurfaces in $\mathbb{R}^{n}$.

Key words: locally quasiconformal mapping, modulus of ring domain

2010 Mathematical Subject Classification: 3062, 3065, 3075, 3080 
1. Let $\Gamma$ be a family of at least locally rectifiable curves in $\mathbb{R}^{n}, n \geq 2$. We remind [1, 2, 3], that modulus of the family of curves $\Gamma$ is defined as

$$
M(\Gamma)=\inf _{\rho} \int_{\mathbb{R}^{n}} \rho^{n}(x) d v_{n},
$$

where $d v_{n}$ is an $n$-dimensional volume element and infimum is taken over all admissible measurable non-negative a. e. functions $\rho$ such that $\int_{\gamma} \rho(x) d s \geq 1$ for any curve $\gamma \in \Gamma$, ds is a differential of arclength on $\gamma$.

The domain $D \subset \mathbb{R}^{n}$ is called ring domain, if its complement consists of exactly two disjointed components of connectivity. Within this article we need not more detailed specification of types of this components because we'll deal with locally quasiconformal images of spherical rings.

Let us consider the family $\Gamma$ of all possible curves $\gamma \subset D$, joining boundary components of ring domain $D$. The modulus of ring domain $D$ is a number

$$
\operatorname{Mod}(D)=\left(\frac{M(\Gamma)}{\omega_{n-1}}\right)^{1 /(1-n)},
$$

where $\omega_{n-1}$ is an area of the hypersphere of radius 1 in the space $\mathbb{R}^{n}$. Particularly, the modulus of the concentric spherical ring $K(r, R)=\{x \in$ $\left.\in \mathbb{R}^{n}: r<|x|<R\right\}$ is equal to

$$
\operatorname{Mod}(K(r, R))=\ln \frac{R}{r} .
$$

This relation and a couple of following properties are available, for example, in 2], chapter 8 , or in 3, chapter 2. As in the well-known particular case $n=2$ (i.e. in the case $D \subset \mathbb{C}$ ) moduli of the ring domains possess the property of monotonicity:

$$
\operatorname{Mod}\left(D_{1}\right) \leq \operatorname{Mod}\left(D_{2}\right), \text { if } D_{1} \subset D_{2},
$$

and also satisfy the inequality

$$
\operatorname{Mod}(D) \geq \sum_{k=1}^{m} \operatorname{Mod}\left(D_{k}\right),
$$

if ring domains $D_{k}$ are pairwise disjoint and domain $D=\operatorname{int}\left\{\cup_{k=1}^{m} \overline{D_{k}}\right\}$ is also a ring domain. Here int $A$ means the interior of a set $A$, and $A$ is the closure of $A$. 
The term of modulus of family of surfaces (see for example [3], chapter 2) is closely associated with notions of modulus of family of curves and ring domains. Let $\Sigma^{k}$ be the family of $k$-dimensional locally Lipschitz hypersurfaces in $\mathbb{R}^{n}$. The modulus of $\Sigma^{k}$ is the value

$$
M\left(\Sigma^{k}\right)=\inf _{\rho} \int_{\mathbb{R}^{n}} \rho^{n}(x) d v_{n},
$$

where the infimum is taken over all admissible measurable non-negative functions $\rho$ such that $\int_{\sigma} \rho^{k}(x) d s_{k} \geq 1$ for any surface $\sigma \in \Sigma^{k}, d s_{k}$ is an element of $k$-dimensional area on the surface $\sigma$.

Let us consider a ring domain $D \subset \mathbb{R}^{n}$ and the family of all possible curves $\Gamma \subset D$, joining boundary components of $D$ as in the definition of modulus of ring domain $D$. Then the modulus $M(\Gamma)$ can be expressed via the modulus of the family $\Sigma$ of all possible $(n-1)$-dimensional hypersurfaces, separating the boundary components of domain $D$ :

$$
M(\Gamma)=\left(\frac{1}{M(\Sigma)}\right)^{n-1}
$$

This result follows from investigation of F. Gehring and W. Ziemer and can be found in 3 . The families $\Gamma$ and $\Sigma$ in this case are called conjugate.

2. A homeomorphism $f$ is called quasiconformal in a domain $D \subset \mathbb{R}^{n}$, if there exists a constant $Q \geq 1$ such that for any family of curves $\Gamma \subset D$

$$
\frac{1}{Q} M(\Gamma) \leq M(f(\Gamma)) \leq Q M(\Gamma) .
$$

There is much literature devoted to the properties of spatial quasiconformal mappings (see for example monographs [2, 3, 1]). The quantity $Q$, as in the planar case, is called a coefficient of quasiconformality of $f$. Hence the quasiinvariance of the moduli of the families of curves is placed in definition (5) of quasiconformal mappings. The mapping $f$ is conformal if and only if $Q=1$. It should be noted that unlike the planar case the class of spatial conformal mappings is very narrow whereas the set of quasiconformal mappings is still wide and useful for many applications also in the case $n>2$.

Every quasiconformal homeomorphism $f$ is differentiable almost everywhere in the domain $D$ and consequently the dilatation of the mapping $f$ (see for example [2]) is defined a.e. as

$$
p_{f}(x)=\max \left\{\frac{L_{f}^{n}(x)}{\left|J_{f}(x)\right|}, \frac{\left|J_{f}(x)\right|}{l_{f}^{n}(x)}\right\},
$$


where $J_{f}(x)$ is the Jacobian of $f$,

$$
l_{f}(x)=\min _{|h|=1}\left|f^{\prime}(x) \cdot h\right|, \quad L_{f}(x)=\max _{|h|=1}\left|f^{\prime}(x) \cdot h\right| .
$$

Here $f^{\prime}(x)$ denotes the Jacobi matrix, i. e., the derivative of function $f$ at point $x$. The coefficient of quasiconformality is connected with dilatation by means of relation

$$
Q=\operatorname{essup}_{x \in D} p_{f}(x)
$$

A homeomorphism $f$ is called locally quasiconformal in the domain $D$, if $f$ is quasiconformal on every compact set $K \subset D$. It is obvious that such mappings can distort the moduli of families of curves indefinitely. Nevertheless as in the planar case [4, 5, 6, it is possible to obtain some useful estimations of distortion of moduli in terms of dilatation majorant of locally quasiconformal mappings.

Consider a locally quasiconformal mapping $f$ of the ring $K(r, R)$ and define for $t \in(r, R)$ the majorant of dilatation of the mapping $f$ in the following way:

$$
P_{f}(t)=\lim _{\varepsilon \rightarrow 0+} \operatorname{essup}\left\{p_{f}(x): t-\varepsilon \leq|x| \leq t+\varepsilon\right\} .
$$

It is obvious that function $P_{f}(t)$ is well defined for all $t \in(r, R)$ and $P_{f}(t) \geq 1$. Moreover, the following theorem is true:

Theorem 1. For any locally quasiconformal mapping $f$ of the ring $K(r, R)$, $0<r<R<\infty$, the function $P_{f}(t)$ is measurable on $(r, R)$.

Proof. Let $f$ satisfy the condition of the theorem. Consider the partition of the segment $[r, R]$ by the points $t_{m, l}=r+(R-r) l / 2^{m}, m \in \mathbb{N}, l=$ $=0, \ldots, 2^{m}$, and define the sequence of functions $P_{f, m}(t)$ on the interval $(r, R)$ such that

$$
P_{f, m}(t)=\left\{\begin{array}{l}
\operatorname{essup}\left\{p_{f}(x): t_{m, l-1} \leq|x| \leq t_{m, l}\right\} \text { for } t \in\left(t_{m, l-1}, t_{m, l}\right] \\
l=1, \ldots, 2^{m}
\end{array}\right.
$$

It is clear that $P_{f, m}(t) \geq 1$ and $P_{f, m}(t)$ is non-increasing in $m$ for any fixed $t \in(r, R)$. Hence there exist the limits

$$
Q_{f}(t)=\lim _{m \rightarrow \infty} P_{f, m}(t), t \in(r, R)
$$


at that function $Q_{f}(t)$ is measurable as the limit of the step-functions (see, for example [7], chapter V, §4).

Let us show that

$$
Q_{f}(t)=P_{f}(t) \text { a.e. on }(r, R) \text {. }
$$

Let $t \neq t_{m, l}$ for any pair $m, l$. Then there exists the sequence of points $t_{m_{k}, l_{k}}$, such that $t \in\left(t_{m_{k}, l_{k}-1}, t_{m_{k}, l_{k}}\right)$ for all $k \in \mathbb{N}$ and $t_{m_{k}, l_{k}} \rightarrow t$ as $k \rightarrow \infty$. Consider $\varepsilon_{k}=\min \left\{t-t_{m_{k}, l_{k}-1}, t_{m_{k}, l_{k}}-t\right\}>0$. Then

$$
\operatorname{essup}\left\{p_{f}(x): t-\varepsilon_{k} \leq|x| \leq t+\varepsilon_{k}\right\} \leq P_{f, m_{k}}(t)
$$

Now passing to the limit as $k \rightarrow \infty$ in the last inequality, we conclude that

$$
P_{f}(t) \leq Q_{f}(t) .
$$

On the other hand, if we choose $\varepsilon_{k}=\max \left\{t-t_{m_{k}, l_{k}-1}, t_{m_{k}, l_{k}}-\right.$ $-t\}+1 / k$, then we obtain, in the similar way, the opposite inequality $P_{f}(t) \geq Q_{f}(t)$. Therefore,

$$
P_{f}(t)=Q_{f}(t)
$$

for all $t \neq t_{m, l}$, i.e. almost everywhere on $(r, R)$. So the function $P_{f}$ is measurable as coincident a.e. with the measurable function $Q_{f}$.

The next theorem allows us to estimate the distortion of the moduli of rings under locally quasiconformal mappings in terms of the dilatation majorant $P_{f}$ of the function $f$. This theorem of course goes back to the famous Belinsky lemma for planar quasiconformal mappings [8. In the case of planar locally quasiconformal mappings corresponding generalization of Belinsky lemma was proved by the author in 2009 [4. Also it should be remarked that the next theorem may be deduced from results recently obtained by M. Brakalova, I. Markina and A. Vasilev 9]. But independent proof proposed in this article differs from the cited above and is shorter and direct. Related results were also considered in [10, 11] for Heisenberg groups and for some special cases for $n=2$.

The sharpness of the estimation in forthcoming theorem 2 is regarded in the sense that there are locally quasiconformal mappings of rings with unbounded and constant on a.e. hyperspheres dilatation for which equalities hold in left or right hand sides of (8). 
Theorem 2. Let $f$ be a locally quasiconformal mapping of the ring $K(r, R), 0<r<R<\infty$, onto the ring domain $D \subset \mathbb{R}^{n}$ and the function $P_{f}(t)$ is defined by equality (6). Then

$$
\int_{r}^{R} P_{f}^{1 /(1-n)}(t) \frac{d t}{t} \leq \operatorname{Mod}(D) \leq \int_{r}^{R} P_{f}^{1 /(n-1)}(t) \frac{d t}{t} .
$$

Both inequalities are sharp.

Proof. Let the conditions of the theorem be fulfilled. In the view of the measurability of the function $P_{f}(t)$ and boundedness of the function $P_{f}^{1 /(1-n)}(t) / t$ on the interval $(r, R)$ for $r>0, R<\infty$, both integrals in (8) exist and integral in the left hand part of this inequality is finite and positive.

a) At first, let us prove the lower estimation. Consider the partition of the segment $[r, R]$ by the points $t_{m, l}=r+(R-r) l / 2^{m}, m \in \mathbb{N}, l=$ $=0, \ldots, 2^{m}$, and represent the ring $K(r, R)$ as the finite union of the rings $K\left(t_{m, l-1}, t_{m, l}\right), l=1, \ldots, 2^{m}$, with the attached appropriate hyperspheres. Then in the view of homeomorphism of $f$, inequality (3) and property (5) of the quasiinvariance of moduli under quasiconformal mapping $f$ of the each of rings $K\left(t_{m, l-1}, t_{m, l}\right), l<2^{m}$, we obtain that

$$
\begin{gathered}
\operatorname{Mod}(D)=\operatorname{Mod}(f(K(r, R)))=\operatorname{Mod}\left(\bigcup_{l=1}^{2^{m}} f\left(K\left(t_{m, l-1}, t_{m, l}\right)\right)\right) \geq \\
\geq \sum_{l=1}^{2^{m}} \operatorname{Mod}\left(f\left(K\left(t_{m, l-1}, t_{m, l}\right)\right)\right) \geq \sum_{l=1}^{2^{m}} P_{f, m}^{1 /(1-n)}\left(t_{m, l}\right) \operatorname{Mod}\left(K\left(t_{m, l-1}, t_{m, l}\right)\right),
\end{gathered}
$$

where $P_{f, m}$ are defined by equalities (7). Taking into account formulae (1) for moduli of rings $K\left(t_{m, l-1}, t_{m, l}\right)$ we obtain

$$
\operatorname{Mod}(D) \geq \sum_{l=1}^{2^{m}} P_{f, m}^{1 /(1-n)}\left(t_{m, l}\right) \ln \frac{t_{m, l}}{t_{m, l-1}}=\int_{r}^{R} P_{f, m}^{1 /(1-n)}(t) \frac{d t}{t} .
$$

Passing to the limit in the integrals of step functions and taking into account that $P_{f, m} \rightarrow P_{f}$ for a.e. $t \in(r, R)$, we arrive to a required inequality in the left side of (8).

b) Now we are going to prove the upper estimation. In the view of definition of the modulus of a ring domain and the property (4), connecting the moduli of the conjugated families of curves and hypersurfaces, we 
have:

$$
\operatorname{Mod}(f(K(r, R)))=\omega_{n-1}^{1 /(n-1)} M(f(\Sigma)) \leq \omega_{n-1}^{1 /(n-1)} \int_{\mathbb{R}^{n}} \rho^{n}(y) d v_{n},
$$

where $\Sigma$ is the family of all $(n-1)$-dimensional hypersurfaces, separating the boundary components of the concentric ring $K(r, R)$, and $\rho(y)$ is an arbitrary measurable non-negative function admissible in the definition of modulus of family $f(\Sigma)$.

As $\rho(y)$ we will consider the function

$$
\rho(y)=\left\{\begin{array}{l}
\left(\rho_{0} / \mu_{n-1}^{1 /(n-1)}\right) \circ f^{-1}(y), \text { for } y \in f(K(r, R)), \\
0, \text { if } y \notin f(K(r, R)),
\end{array}\right.
$$

where $\rho_{0}$ is an arbitrary admissible function for a modulus of family $\Sigma$ of $(n-1)$-dimensional hypersurfaces, $\mu_{n-1}(x)$ is the minimal distortion of the $(n-1)$-dimensional volume at the point $x$ under the mapping $f$. Defined in this way, function $\rho$ is admissible in the problem of finding the modulus of the family of surfaces $f(\Sigma)$. Indeed, for any surface $\sigma \in \Sigma$ and its image $f(\sigma)$ let $d s_{n-1}$ and $d s_{n-1}^{*}$ be elements of $(n-1)$-dimensional area correspondingly. Then we obtain

$$
\begin{aligned}
\int_{f(\sigma)} \rho^{n-1}(y) d s_{n-1}^{*} & \geq \int_{\sigma}\left(\frac{\rho_{0}(x)}{\mu_{n-1}^{1 /(n-1)}(x)}\right)^{n-1} \mu_{n-1}(x) d s_{n-1}= \\
& =\int_{\sigma} \rho_{0}^{n-1}(x) d s_{n-1} \geq 1 .
\end{aligned}
$$

Note that for almost all $y$ the preimage of the infinitesimal ball $\{y \in$ $\in f(K(r, R)):|y-f(x)| \leq \delta\}$ of radius $\delta$ under the mapping $f$ is a domain, close to ellipsoid with semiaxes $a_{1} \geq a_{2} \geq \ldots \geq a_{n}$, where

$$
a_{1}=\frac{\delta}{l_{f}(x)}, a_{n}=\frac{\delta}{L_{f}(x)}
$$

Then

$$
\mu_{n-1}(x)=\frac{\delta^{n-1}}{a_{1} a_{2} \cdots a_{n-1}}, \text { whereas } J_{f}(x)=\frac{\delta^{n}}{a_{1} a_{2} \cdots a_{n-1} a_{n}} .
$$


Using (10) and the latter equality, we obtain

$$
\begin{aligned}
& \frac{J_{f}(x)}{\mu_{n-1}^{n /(n-1)}(x)}=\frac{\left(a_{1} a_{2} \cdots a_{n-1}\right)^{n /(n-1)}}{a_{1} a_{2} \cdots a_{n-1} a_{n}}= \\
= & \left(\frac{a_{1} a_{2} \cdots a_{n}}{a_{n}^{n}}\right)^{1 /(n-1)}=\left(\frac{L_{f}^{n}(x)}{J_{f}(x)}\right)^{1 /(n-1)} .
\end{aligned}
$$

After the change of variables under the sign of integral in the right hand side of (9), in view of equality (11) we obtain the estimations

$$
\begin{aligned}
\operatorname{Mod}( & f(K(r, R))) \leq \omega_{n-1}^{1 /(n-1)} \int_{K(r, R)} \rho_{0}^{n}(x) \frac{J_{f}(x)}{\mu_{n-1}^{n /(n-1)}(x)} d v_{n}= \\
& =\omega_{n-1}^{1 /(n-1)} \int_{K(r, R)} \rho_{0}^{n}(x)\left(\frac{L_{f}^{n}(x)}{J_{f}(x)}\right)^{1 /(n-1)} d v_{n} \leq \\
& \leq \omega_{n-1}^{1 /(n-1)} \sum_{l=1}^{2^{m}} \int_{K\left(t_{m, l-1}, t_{m, l}\right)} \rho_{0}^{n}(x) p_{f}^{1 /(n-1)}(x) d v_{n} \leq \\
& \leq \omega_{n-1}^{1 /(n-1)} \sum_{l=1}^{2^{m}} P_{f, m}^{1 /(n-1)}\left(t_{m, l}\right) \int_{K\left(t_{m, l-1}, t_{m, l}\right)} \rho_{0}^{n}(x) d v_{n},
\end{aligned}
$$

where the rings $K\left(t_{m, l-1}, t_{m, l}\right)$ are the same that in the part a) of the proof of this theorem, and the functions $P_{f, m}$ are defined via equality (6).

It remains to note that function $\rho_{0}(x)=\left(\omega_{n-1}^{1 /(n-1)}|x|\right)^{-1}$ is admissible in the problem of finding of the modulus of family $\Sigma$ in the ring $K(r, R)$ and after the changeover to the spherical coordinates

$$
\int_{K\left(t_{m, l-1}, t_{m, l}\right)} \rho_{0}^{n}(x) d v_{n}=\frac{1}{\omega_{n-1}^{n /(n-1)}} \int_{t_{m, l-1}}^{t_{m, l}} \frac{d t}{t} \int_{|x|=1} d s_{n-1}=\frac{1}{\omega_{n-1}^{1 /(n-1)}} \int_{t_{m, l-1}}^{t_{m, l}} \frac{d t}{t} .
$$

Taking into account the last equalities we obtain from (12) that

$$
\operatorname{Mod}(f(K(r, R))) \leq \sum_{l=1}^{2^{m}} P_{f, m}^{1 /(n-1)}\left(t_{m, l}\right) \int_{t_{m, l-1}}^{t_{m, l}} \frac{d t}{t}=\int_{r}^{R} P_{f, m}^{1 /(n-1)}(t) \frac{d t}{t} .
$$

Passing to the limit in the integrals of the step-functions as in the part a) we obtain the desired upper estimation in (8). 
c) In order to prove the sharpness of inequalities (8) we consider the sequence of points $r_{k}=1-1 / 2^{k}, k=0,1,2, \ldots$. For each $k$ we define the quasiconformal homeomorphism of the set $K_{k}:=\left\{x \in \mathbb{R}^{n}: r_{k}<|x| \leq\right.$ $\left.\leq r_{k+1}\right\}$ by the formulae

$$
f_{k}(x)=x \frac{|x|^{\alpha_{k}-1}}{c_{k}}
$$

where exponents $\alpha_{k}:=2^{-k} \rightarrow 0$ as $k \rightarrow \infty$, and coefficients $c_{k}$ are defined by recurrent ratios

$$
c_{0}=1, c_{k+1}=c_{k} r_{k+1}^{\alpha_{k+1}-\alpha_{k}}
$$

Then the function

$$
f(x)=\left\{\begin{array}{l}
f_{k}(x), \text { if } x \in K_{k}, \\
k=0,1,2, \ldots
\end{array}\right.
$$

is a locally quasiconformal homeomorphism of the unit ball $\mathbf{B}^{n}=\{x \in$ $\left.\in \mathbb{R}^{n}:|x|<1\right\}$ onto the ball $B(R)=\left\{x \in \mathbb{R}^{n}:|x|<R\right\}$ with radius

$$
R=\lim _{k \rightarrow \infty} \frac{r_{k+1}^{\alpha_{k}}}{c_{k}}=r_{1} \prod_{k=1}^{\infty}\left(\frac{r_{k+1}}{r_{k}}\right)^{\alpha_{k}}<\infty .
$$

Here the convergence of the infinite product follows from the inequality $r_{k+1} / r_{k} \leq 3 / 2$ for any $k \geq 1$ and from the fact that $\ln R$ is majorized by the converging series

$$
\ln r_{1}+\ln 3 / 2 \sum_{k=1}^{\infty} \alpha_{k}=\ln r_{1}+\ln 3 / 2 \sum_{k=1}^{\infty} 2^{-k} .
$$

The mapping (13) is identical in the neighborhood of the origin and maps the ring $K\left(r_{1}, 1\right)$ locally quasiconformally onto the ring $K\left(r_{1}, R\right)$. With a glance of formulae (1) for a moduli of rings

$$
\operatorname{Mod}\left(f\left(K\left(r_{1}, 1\right)\right)=\operatorname{Mod}\left(K\left(r_{1}, R\right)\right)=\ln \frac{R}{r_{1}} .\right.
$$

The function $f$ maps each ring $K_{k}$ in one-to-one manner onto the rings $\left\{x \in \mathbb{R}^{n}: r_{k}^{\alpha_{k}} / c_{k}<|x| \leq r_{k+1}^{\alpha_{k}} / c_{k}\right\}$. The mapping $f$, inside every ring $K_{k}$, is quasiconformal and is known as the radial mapping. It is possible to calculate (see, for example, [1], chapter 16, example 16.2, or [3], chapter 4, $\S 2,4)$ that in $K_{k}$ the radial mapping $f$ has a constant dilatation $p_{f}(x)=$ $=\alpha_{k}^{1-n}=2^{k(n-1)}$. Therefore $P_{f}(t)=\alpha_{k}^{1-n}$ for any $t \in\left(r_{k}, r_{k+1}\right), k \geq 1$. 
In such a way

$$
\int_{r_{1}}^{1} P_{f}^{1 /(1-n)}(t) \frac{d t}{t}=\sum_{k=1}^{\infty} \alpha_{k} \int_{r_{k}}^{r_{k+1}} \frac{d t}{t}=\sum_{k=1}^{\infty} \alpha_{k} \ln \frac{r_{k+1}}{r_{k}}=\ln R-\ln r_{1},
$$

i.e. $\operatorname{Mod}\left(f\left(K\left(r_{1}, 1\right)\right)=\int_{r_{1}}^{1} P_{f}^{1 /(1-n)}(t) \frac{d t}{t}\right.$. The sharpness of the lower estimation in inequality (8) has been proved.

In order to prove the sharpness of the upper estimation in (8) it is sufficient to change the exponents $\alpha_{k}$ in the definition of function (13) putting, for example, $\alpha_{k}=(3 / 2)^{k}$. Then the radius $R$, determined by (13), is finite as before since, in the view of relation $\ln \left(r_{k+1} / r_{k}\right) \approx \ln \left(1+2^{-k}\right) \approx$ $\approx 2^{-k}$, the series

$$
\ln R=\ln r_{1}+\sum_{k=1}^{\infty} \alpha_{k} \ln \frac{r_{k+1}}{r_{k}}
$$

has the same asymptotic as the converging series $\sum_{k=1}^{\infty}(3 / 4)^{k}$. In addition, the dilatation of the mapping $f$ in every $\operatorname{ring} K_{k}$ is equal to $p_{f}(x)=$ $=\alpha_{k}^{n-1}=(3 / 2)^{k(n-1)}$ and

$$
\int_{r_{1}}^{1} P_{f}^{1 /(n-1)}(t) \frac{d t}{t}=\sum_{k=1}^{\infty} \alpha_{k} \ln \frac{r_{k+1}}{r_{k}}=\ln R-\ln r_{1}=\operatorname{Mod}\left(f\left(K\left(r_{1}, 1\right)\right)\right),
$$

that illustrates the sharpness of the upper estimation in (8).

Remark. If the mapping $f$ in theorem 2 is $Q$-quasiconformal, then $P_{f}(t) \leq$ $\leq Q$ and the classical estimations (5) of the distortion of moduli follow easily from inequalities (8).

3. In this section we will apply theorem 2 to the proof of the estimation of the derivative of locally quasiconformal automorphism of the ball $\mathbf{B}^{n}=$ $=\left\{x \in \mathbb{R}^{n}:|x|<1\right\}$.

Theorem 3. Let $f$ be a locally quasiconformal automorphism of the unit ball $\boldsymbol{B}^{n}$ such that $f(0)=0$, function $f$ be differentiable at the origin and $l_{f}(0)=L_{f}(0)=:\left|f^{\prime}(0)\right|$, i. e. $f$ be conformal at the point $x=0$. Then

$$
\int_{0}^{1}\left(1-P_{f}^{1 /(n-1)}(t)\right) \frac{d t}{t} \leq \ln \left|f^{\prime}(0)\right| \leq \int_{0}^{1}\left(1-P_{f}^{1 /(1-n)}(t)\right) \frac{d t}{t} .
$$

Estimations are sharp. 
Proof. Let the conditions of the theorem be fulfilled. Consider the small value $\varepsilon>0$. Function $f$ maps the ring $K(\varepsilon, 1)$ onto ring domain $D_{\varepsilon}$, whose outer boundary component coincides with the unit hypersphere $\mathbf{S}^{n-1}=\left\{x \in \mathbb{R}^{n}:|x|=1\right\}$, whereas inner boundary component, in view of conformality of $f$ at origin, coincides accurate to $o(\varepsilon)$ with the hypersphere of radius $\varepsilon\left|f^{\prime}(0)\right|$ with the center at point $x=0$.

First we prove the upper estimation in (15). In view of property (2) of monotonicity of modulus of ring domains

$$
\operatorname{Mod}\left(D_{\varepsilon}\right) \leq \operatorname{Mod}\left(K\left(\varepsilon\left|f^{\prime}(0)\right|-o(\varepsilon), 1\right)\right)=-\ln \left(\varepsilon\left|f^{\prime}(0)\right|-o(\varepsilon)\right)
$$

for any small $\varepsilon>0$. Here $o(\varepsilon)$ is some positive infinitesimal function that decreases faster than $\varepsilon$ when $\varepsilon \rightarrow 0+$.

From the other side in view of theorem 2

$$
\operatorname{Mod}\left(D_{\varepsilon}\right) \geq \int_{\varepsilon}^{1} P_{f}^{1 /(1-n)}(t) \frac{d t}{t} .
$$

Therefore,

$$
-\ln \left(\varepsilon\left|f^{\prime}(0)\right|-o(\varepsilon)\right) \geq \int_{\varepsilon}^{1} P_{f}^{1 /(1-n)}(t) \frac{d t}{t},
$$

i.e.

$$
\ln \left|f^{\prime}(0)\right| \leq-\int_{\varepsilon}^{1}\left(P_{f}^{1 /(1-n)}(t)-1\right) \frac{d t}{t}+o(1),
$$

Passing to the limit as $\varepsilon \rightarrow 0$, we obtain the required upper estimation in $(15)$.

The proof of the lower estimation may be obtained in the similar way with the help of upper estimation in (8). Indeed, there exists a positive infinitesimal function $o(\varepsilon)$ such that $K\left(\varepsilon\left|f^{\prime}(0)\right|+o(\varepsilon), 1\right) \subset D_{\varepsilon}$ and in view of $(1),(2),(8)$

$$
\begin{gathered}
\ln \frac{1}{\left|f^{\prime}(0)\right|}+\ln \frac{1}{\varepsilon}-o(1)=\ln \frac{1}{\varepsilon\left|f^{\prime}(0)\right|+o(\varepsilon)}= \\
=\operatorname{Mod}\left(K\left(\varepsilon\left|f^{\prime}(0)\right|+o(\varepsilon), 1\right)\right) \leq \operatorname{Mod}\left(D_{\varepsilon}\right) \leq \int_{\varepsilon}^{1} P_{f}^{1 /(n-1)}(t) \frac{d t}{t} .
\end{gathered}
$$

The required lower estimation of $\left|f^{\prime}(0)\right|$ follows from this relationship.

In order to prove the sharpness of the inequality (15) we consider the function $F(x)=f(x) / R$, where $f, R$ are determined by equalities (13) 
and (14) respectively. The mapping $F$ is a locally quasiconformal automorphism of the ball $\mathbf{B}^{n}$, having in the neighborhood of the origin representation $F(x)=x / R$. Hence $f^{\prime}(0)=I / R$, where $I$ is a Jacobi matrix of the identical transformation and $\left|F^{\prime}(0)\right|=l_{F}(0)=L_{F}(0)=1 / R$.

From the other side, if $\alpha_{k}=2^{-k}$ then, as was mentioned above, $P_{F}(t)=\alpha_{k}^{1-n}$ for any $t \in\left(r_{k}, r_{k+1}\right), k \geq 1$, and $P_{F}(t)=1$ for all $t \in\left[0, r_{1}\right)$. Therefore

$$
\begin{aligned}
& \int_{0}^{1}\left(P_{F}^{1 /(1-n)}(t)-1\right) \frac{d t}{t}=\sum_{k=1}^{\infty}\left(\alpha_{k}-1\right) \int_{r_{k}}^{r_{k+1}} \frac{d t}{t}= \\
& =\sum_{k=1}^{\infty} \alpha_{k} \ln \frac{r_{k+1}}{r_{k}}+\ln r_{1}=\ln R .
\end{aligned}
$$

Consequently

$$
\ln \left|F^{\prime}(0)\right|=\ln \frac{1}{R}=-\int_{0}^{1}\left(P_{F}^{1 /(1-n)}(t)-1\right) \frac{d t}{t},
$$

and the sharpness of the upper estimation of $\left|f^{\prime}(0)\right|$ is proved.

The sharpness of the lower estimation in inequality (15) may be demonstrated in a similar way if we choose the values $\alpha_{k}=(3 / 2)^{k}$.

Remark. Note that the convergence of the integrals in inequalities (15) depends on the velocity of tending of function $P_{f}(t)$ to 1 as $t \rightarrow 0+$ and on the velocity of growth of $P_{f}(t)$ as $t \rightarrow 1-$. In particular case the values $\left|f^{\prime}(0)\right|$ are bounded and separated from zero, if there exist positive numbers $\alpha$ and $\beta$ such that

$$
\left\{\begin{array}{l}
P_{f}(t)-1=o\left(t^{\alpha}\right) \text { if } t \rightarrow 0+ \\
P_{f}(t)(1-t)^{n-1}=o\left((1-t)^{\beta}\right) \text { if } t \rightarrow 1-.
\end{array}\right.
$$

For example, if in the assumption of theorem 3 the majorant $P_{f}(t) \leq$ $\leq((1+t) /(1-t))^{n-1}$, then $\left|f^{\prime}(0)\right| \leq 4$, but in the case of $P_{f}(t) \geq(1-$ $-1 / \ln t)^{n-1}$ we have only the trivial estimation $\left|f^{\prime}(0)\right| \leq \infty$.

Acknowledgment. The research was supported by the Russian Foundation for Basic Research (project 14-01-92692). 


\section{References}

[1] Väisälä J. Lectures on n-dimensional quasiconformal mappings. SpringerVerlag, Berlin, Heidelberg, NY, 1971.

[2] Anderson G. D., Vamanamurthy M. K., Vuorinen M. Conformal invariants, inequalities an quasiconformal maps. New York, 1997.

[3] Sychev A. V. Spatial quasiconformal mappings. Novosibirsk, 1975. (in Russian)

[4] Graf S. Yu., Eyelangoli O. R. On the distortion of the moduli of the double connected domains under locally quasiconformal mappings. Application of the functional analysis in the theory of approximation. Tver State University, 2009, pp. 34-43. (in Russian)

[5] Graf S. Yu. The growth theorems in the classes of normalized locally quasiconformal mappings. Probl. Anal. Issues Anal., 2013, vol. 2(20), no. 2, pp. 3-20. (in Russian) DOI: 10.15393/j3.art.2013.2381.

[6] Graf S. Yu. An analogue of the Schwarz lemma for locally quasiconformal automorphisms of the unit disk. Russian Mathematics, 2014, vol. 58, no. 11, pp. 74-79.

[7] Kolmogorov A. N., Fomin S. V. Elements of the function theory and functional analysis. Moscow, 1989. (in Russian)

[8] Belinsky P. P. General properties of quasiconformal mappings. Novosibirsk, 1974. (in Russian)

[9] Brakalova M., Markina I., Vasilev A. Extremal functions for modules of systems of measures. arXiv:1409.1626v2 [math.CA], 15 Sep. 2014, 49 pp.

[10] Balogh Z. M., Fässler K., Platis I. D. Modulus of curve families and extremality of spiral-stretch maps. Journal d'analyse mathématique, 2011, vol. 113, pp. 265-291. DOI: 10.1007/s11854-011-0007-x.

[11] Balogh Z. M., Fässler K., Platis I. D. Modulus method and radial stretch map in the Heisenberg group. Ann. Acad. Sci. Fenn., Mathematica, vol. 38, 2013, pp. 149-180. DOI: 10.5186/aasfm.2013.3811.

Received July 11, 2015.

In revised form, November 17, 2015.

Tver State University

33, Zheliabova st., 170100 Tver, Russia;

Petrozavodsk State University

33, Lenina st., 185910 Petrozavodsk, Russia

E-mail: Sergey.Graf@tversu.ru 